\title{
DOUBLE-BILAYER: A NEW PHASE FORMED BY LYSOPHOSPHOLIPIDS AND THE CORRESPONDING FATTY ACID
}

\author{
Sérgio S. Funari*\# \\ EMBL Outstation Hamburg, DESY \\ Gert Rapp \#\# \\ EMBL Outstation Hamburg, DESY \\ Frank Richter \\ EMBL Outstation Hamburg, DESY, Dept. of Physics, E22 Biophysics, TU Munich, D-85748 Garching, Germany
}

Recebido em 23/4/08; aceito em 31/10/08; publicado na web em 6/3/09

\begin{abstract}
The product of catalytic activity of the enzyme phospholipase A2, which resembles the core unit of animal toxins, on phospholipids is a 1:1 mixture of lysolipid and fatty acid. This mixture was studied by time-resolved simultaneous small- and wide angle $\mathrm{x}$-ray diffraction over the temperature range from 23 to $53.5^{\circ} \mathrm{C}$. An unusually large lamellar structure was observed, with $d=11 \mathrm{~nm}$, contradicting the complex functional dimer model between lysolipid and fatty acid. It can be explained by formation of a "doublebilayer", a new phase consisting of two different bilayers, one formed by lysophospholipid and other by fatty acid, bound together by head group interactions. Its strucutre was confirmed by simulations of the X-ray scattering pattern.
\end{abstract}

Keywords: PLA 2 lipid bilayer; X-rays.

\section{INTRODUCTION}

Cell membranes are of crucial importance by providing the structural basis and the boundary for the cells. Their main components are functional proteins and lipids that self-assemble into bilayers where other components, as e.g. cholesterol, are incorporated. ${ }^{1}$ The membrane encapsulates important molecules such as DNA and RNA and protects them from foreign agents that could damage the cell components. On the other hand, the membrane must be permeable to nutrients and to the release of (toxic) products of its metabolism. Their remarkable properties are prime subjects in disease and drug therapy. ${ }^{2}$

Secretory phospholipase $\mathrm{A}_{2}$-sPLA2- has been successfully used to release drugs incorporated into degradable liposomes. It was shown that when loaded with doxorubicin (active agent in DOXIL ${ }^{\circledR}$ ) these liposomes led a similar uptake of it into HT-29 colon cancer cells, compared to the drug as free doxorubicin. Interestingly, a similar experiment using cisplatin showed that the loaded liposomes were more cytotoxic than free cisplatin. This has been attributed to a possible additive effect of the sPLA2 catalyzed hydrolysis products to membrane perturbation, illustrating the importance of understanding the effect of these products on the cell membrane. ${ }^{3}$

Here we are concerned with the products of phospholipid degradation, as in the case of their metabolism. The enzyme phospholipase $\mathrm{A}_{2}\left(\mathrm{PLA}_{2}\right)$ hydrolyses the ester bond of glycerol phospholipids at the $\mathrm{C}_{2}$ carbon, therefore its activity on dipalmitoylphosphatidylcholine (DPPC) produces palmitoyl-lyso-phosphatidyl choline (LPC) and palmitic acid (PA), in a 1:1 molar ratio. Both products are known as second messengers in a number of signal transduction pathways and LPC is also a powerful detergent. Further, the enzyme PLA is the basic active unit of toxins found in animal venoms. ${ }^{4}$ Therefore one

*e-mail: sergio.funari@ desy.de

"Present address: HASYLAB at DESY, D-22607, Notkestr. 85, Hamburg, Germany.

"\#Present address: Rapp OptoElectronic, Gehlenkamp 9a, 22559 Hamburg, Germany could look for correlations between the structure of the products and symptoms occurring in persons or animals poisoned by snake bites or bee stings. Whereas there is agreement on the actual binding site and the catalytic mechanism of PLA 2 activity, ${ }^{5,6}$ there is not much known about the structural behaviour of the reaction products upon their formation.

Many PLA2s show high sensitivity to surface topology, with regions of high curvature stimulating hydrolysis, despite displacing water from the lipid surface upon binding. The interface recognition is mediated by both electrostatic and hydrophobic components. PLA2 shows high activity toward zwitterionic phosphocholines, due to hydrophobic interactions and "both degradation products favour nonplanar geometries, namely normal micelles (lysophospholipids) and inverted hexagonal phases (fatty acids)". ${ }^{7}$

Differential Scanning Calorimetry -DSC- thermograms of our target system, LPC/PA 1:1 mixtures, using scan rates of $0.2^{\circ} \mathrm{C} / \mathrm{min}$ showed two endothermic transitions; one at about 42 and another at $48{ }^{\circ} \mathrm{C}$ (ref. 8 and Figure 1). On cooling one observes a different thermogram, resolving a well defined transition at $46.8^{\circ} \mathrm{C}$ followed by a very broad transition spanned over ca. $15^{\circ} \mathrm{C}$. The phase transitions observed on heating and cooling are not in complete agreement with the previous DSC features of Jain and also do not agree either with each of the single components or with DPPC in excess water. ${ }^{9,10}$

Others have employed electron and polarising optical microscopies, as well as ${ }^{31} \mathrm{PNMR}$, to investigate such mixtures. The data reported led to a bilayer structure. ${ }^{9,11}$ Those results were interpreted in terms of formation of a functional dimer that consists of 1:1 LPC/PA mimicking the DPPC phospholipid molecule, ${ }^{8,9}$ despite the headgroup of the lysolipid being much less constrained than the head group of the phosphatidyl lipid. ${ }^{11}$

\section{EXPERIMENTAL}

The amphiphiles were obtained from Avanti Polar Lipids Inc., Alabster, AL, USA. Samples were prepared by dissolving equimolar amounts LPC and PA in a Hepes buffer ( $150 \mathrm{mM} \mathrm{NaCl}, 1 \mathrm{mM} \mathrm{NaN}_{3}$, $1 \mathrm{mM}$ EDTA, and $100 \mathrm{mM}$ Hepes ( $\mathrm{pH} 8)$ in milli-Q-water), to ob- 

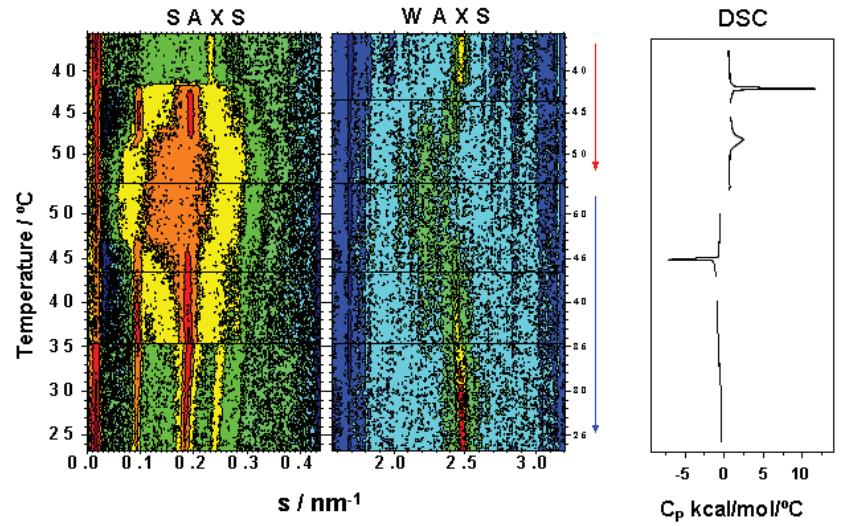

Figure 1. Contour plot of the time-resolved diffraction patterns and DSC from LPC/PA 1:1 molar mixture upon heating and cooling from 23.3 to $53.5^{\circ} \mathrm{C}$ at $0.2^{\circ} \mathrm{C} /$ min. At high temperatures one finds an isotropic phase that upon cooling forms the double-bilayer $L_{b \beta b \beta}$ and $L_{b \beta i \beta,}$, with lattice parameters 10.9 and $8.49 \mathrm{~nm}$ respectively. The horizontal lines indicate temperatures at which the scan was halted to equilibrate the system for half an hour. Right: thermogram showing the different behaviour on heating and cooling, redraw from ref. 8. Note the broad transition on cooling

tain a concentration of (LPC $+\mathrm{PA}) 0.2 \mathrm{M}$. The $\mathrm{NaCl}$ concentration simulates physiological conditions and screens possible Coulombic forces caused by the ionisation of PA molecules at $\mathrm{pH}$ 8. The x-ray measurements were performed at beamline X13 of EMBL at DESY, Hamburg. ${ }^{12,13}$ The following protocol was used: Half an hour equilibration at $35.5^{\circ} \mathrm{C}$, heating to $43.5^{\circ} \mathrm{C}$ at a rate of $0.2^{\circ} \mathrm{C} / \mathrm{min}$, equilibration for half an hour, heating at $0.2{ }^{\circ} \mathrm{C} / \mathrm{min}$ to $53.5^{\circ} \mathrm{C}$, followed by the same protocol in reverse order on the cooling scan. The cooling was further extended to $23.3{ }^{\circ} \mathrm{C}$, followed by a heating back to 35.5 ${ }^{\circ} \mathrm{C}$ (data not shown) to complete the thermocycle.

\section{RESULTS}

In order to identify thermally induced structural changes provoked on the products of PLA 2 activity on DPPC, time-resolved simultaneous small- and wide angle $\mathrm{x}$-ray diffraction (SAXS and WAXS) measurements were performed using the same rate of temperature change as in the DSC by Lemmich et al. ${ }^{8}$

$\mathrm{X}$-ray patterns collected on repeated heating and cooling scans gave qualitatively the same results, enabling us to check the reproducibility of our results and to identify the phase transitions, the structures formed and their lattice parameters.

The main results obtained are an unusually large lamellar lattice dimension of $11 \mathrm{~nm}$ with a second order diffraction peak stronger than the first and only a weak chain's reflection appearing in the WAXS region of the scattering pattern. The formation of this structure could be seen following the strucutral evolution of the system upon a thermal scan. Initially, heating the LPC/PA 1:1 mixture from 37 up to $42{ }^{\circ} \mathrm{C}$, we observe a weak SAXS-reflection corresponding to $4.4 \mathrm{~nm}$ accompanied by a WAXS signal typical of ordered chains, a peak at $s=1 / 0.41 \mathrm{~nm}^{-1}$ characterizing a $\mathrm{L}_{\beta}$ phase. Above $42{ }^{\circ} \mathrm{C}$, in a narrow temperature range $\left(\sim 1^{\circ} \mathrm{C}\right)$, a sharp phase transition takes place, with the SAXS reflections associated with the $\mathrm{L}_{\beta}$ phase vanishing and new lamellar reflections, with corresponding lattice parameter $10.9 \mathrm{~nm}$ appearing, Figure 1. Simultaneously the intensity from the WAXS chains reflection decreases strongly. We shall refer to this rather large structure as double bilayer $\mathrm{L}_{\mathrm{b} \beta \mathrm{b} \beta} .{ }^{14}$

It is noteworthy that this happens at about the chain melting temperature of DPPC $\left(41.5^{\circ} \mathrm{C}\right.$, Lipidat $) .{ }^{15} \mathrm{Also}$, the transition enthalpy for the LPC/PA system, $5.7 \mathrm{Kcal} / \mathrm{mol}$ (Jain et al. ${ }^{9}$ ) is smaller than for DPPC, $8.7 \mathrm{Kcal} / \mathrm{mol}$ (Boyanov et al. ${ }^{16}$ ). This reflects a weaker interaction between the paraffinic chains in this large structure (the double bilayer) than in the corresponding phospholipid, with the phase transition caused by a thermally enhanced chain dynamics; therefore the weak signal appearing on the WAXS region of the scattering patterns (Figure 1).

Further heating to temperatures above $48{ }^{\circ} \mathrm{C}$ results in a phase transition to a single isotropic phase, evidenced by the absence of diffraction peaks (or reflexions) and an increase in scattered intensity, Figures 1 and 2. Attempts to fit the scattering curve to that for isolated micelles of different shapes failed. In order to unequivocally determine the existance of micelles and their respective size and shape requires the determination of the structure factor, due to strong interaction between the units. This is beyond the scope of this article, whose main aim is to describe the large lamellar phases observed.

On Figures 1 to 3, one should note that the apparent peak at very small angles, $\mathrm{s} \sim 0.016 \mathrm{~nm}^{-1}$, is in fact part of the direct beam (the innermost part has been covered by the beam stop), therefore it is not due to the sample, but rather to the instrument.

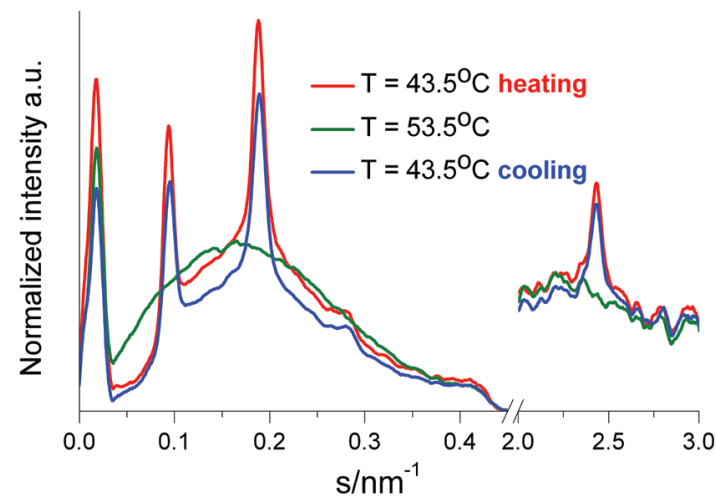

Figure 2. Experimental diffraction patterns observed at temperatures as indicated. The broad scattering pattern corresponds to the isotropic phase. The data at $43.5^{\circ} \mathrm{C}$ (heating red and blue curve) have been multiplied by 1.2, for clarity. The peak at very low s values is the outer part of the direct beam

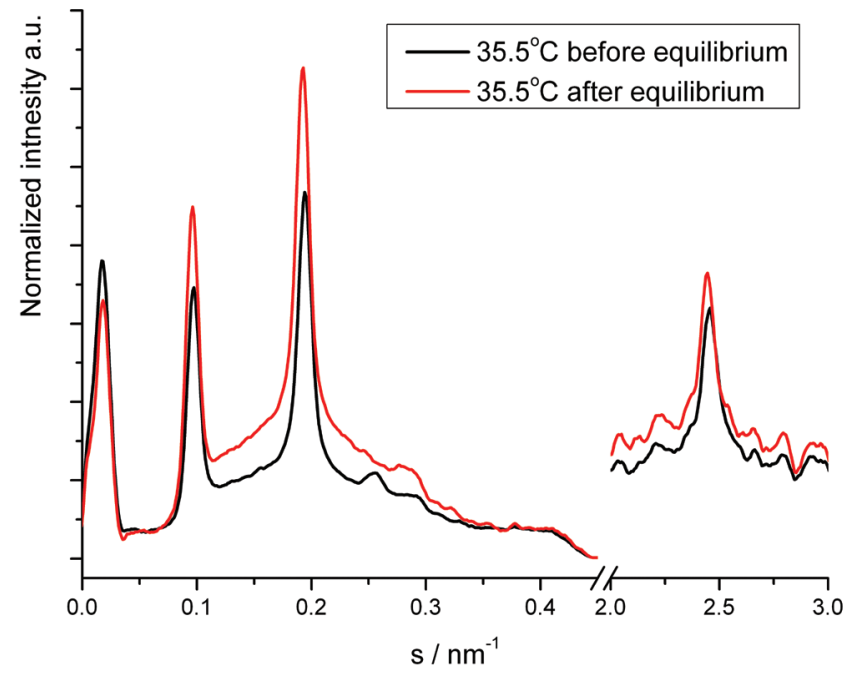

Figure 3. Diffraction patterns illustrating the improvement of resolution upon holding the sample at constant temperature $\left(35.5^{\circ} \mathrm{C}\right)$ for $30 \mathrm{~min}$. The data at $35.5^{\circ} \mathrm{C}$ before equilibrium (red curve) has been multiplied by 1.2, for clarity 


\section{The cooling scan}

On cooling, the isotropic-lamellar phase transition occurs around $47.4{ }^{\circ} \mathrm{C}$ giving rise to this large-spaced lamellar phase $\left(\mathrm{L}_{\mathrm{b} \beta \mathrm{b} \beta}\right)$ with SAXS-reflections slightly less intense than on heating, Figures 1 and 2. This reflects the time required for the system to reorganize enough to show long range order correlation. Upon further cooling, at $37.5^{\circ} \mathrm{C}$ a very weak reflection develops indicating the formation of a transient and metastable phase with a reflection at $s=1 / d=0.12=1 / 8.5 \mathrm{~nm}^{-1}$ co-existing with the initial ones Figure 1. This phase is observed over only a short temperature range and vanishes at $35.5^{\circ} \mathrm{C}$. Note that the lattice parameter of such phase is also rather large, but not as much as the $\mathrm{L}_{\mathrm{b} \beta \mathrm{b} \beta}$ and we shall refer to it also as a double-bilayer, denoted $\mathrm{L}_{\mathrm{b} \beta \mathrm{i} \beta}{ }^{17}$ Simultaneously we observe an intensifying of the the WAXS reflection, associated with organization of the carbon chains, in agreement with the DSC trace of Figure 1. Stopping the cooling scan at $35.5^{\circ} \mathrm{C}$ for $30 \mathrm{~min}$ shows that this transient phase vanishes, giving rise to the gel phase with lattice parameter $4.2 \mathrm{~nm}, s=0.24 \mathrm{~nm}^{-1}$ (Figure 3). The lattice parameters of the remaining phase $\left(\mathrm{L}_{\mathrm{b} \beta \mathrm{b} \beta}\right.$ double-bilayer) and the gel lamellar structure increase slightly on further cooling.

\section{X-ray diffraction pattern simulation}

A successful attempt to understand the structure of such large lamellar phases was to propose some models and calculate their repective SAXS patterns. We simulated the intensity profile of the SAXS reflections for different configurations of the molecules assembly (Figure 4) and checked against the experimental patterns. We modelled the structure factor as described in Nallet et al. and took into account three possibilities, ${ }^{18}$ Figure 4I) single bilayers (dimers of LPC and PA intercalated) composed of two opposed monolayers and a large interlamellar water layer; II) a double bilayer formed such that identical headgroups face each other and III) the double bilayer with the choline and carboxyl head groups facing each other.

We simulated the scattering patterns from the three models proposed in Figure 4, using the box approximation, ${ }^{18}$ aiming to reproduce the relative intensities of the observed reflections (Figure

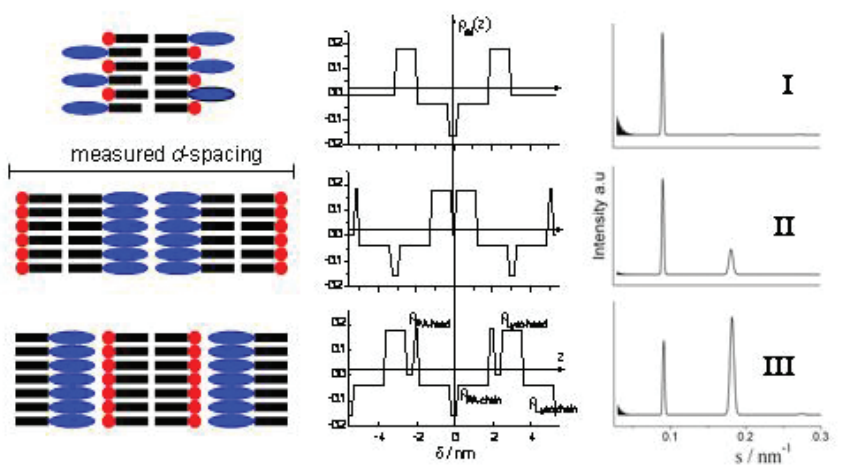

Figure 4. Left: Models for the structure of the lamellar phases formed by a LPC/PA 1:1 mixture. a) single bilayers (dimers of LPC and PA intercalated) composed of two identical monolayers and a large interlamellar water layer; b) a double-bilayer formed such that identical headgroups face each other and c) the double-bilayer consisting of two distinct gel bilayers (LPC-elongated headgroup and PA-spherical headgroup) in direct contact. The simulations show that only model c can account for the relative intensities of the $x$-ray scattering patterns measured, see Figure 4III. Middle: The corresponding electron densities for the layered structures seen on the left, using the box model approximation. Right: Calculated small-angle $x$-ray diffraction patterns for the three possible topological molecular arrangements introduced in Figure 3. Only model III has a pattern compatible with the experimental one, i.e. the second order reflection more intense than the first order
2). The different molecular configurations result in different electron density distributions.

The scattered intensity $I\left(q_{z}\right)$ is given by:

$$
I\left(q_{z}\right) \sim \frac{N}{q_{z}^{2}} P\left(q_{z}\right) S\left(q_{z}\right) \quad q_{z}=\frac{4 \pi \sin \theta}{\lambda}=\frac{2 \pi}{d}=2 \pi s
$$

$\mathrm{N}$ : number of unit cells within the coherently scattering membrane stack; $P\left(q_{z}\right)$ : form factor along the membrane normal; $S\left(q_{z}\right)$ : normalized structure factor of the stack, $2 \theta$ the Bragg angle and $\lambda$ the $\mathrm{x}$-ray wavelength.

The normalized structure factor reads:

$$
S\left(q_{z}\right)=1+2 \sum_{n=1}^{N-1}\left(1-\frac{n}{N}\right) \cos \left(n q_{z} d\right) \exp \left(\frac{-q_{z}^{2}}{2}\left\langle\left(u_{n}-u_{o}\right)^{2}\right\rangle\right)
$$

The trigonometric term within the summation describes the constructive interference from the $N$ unit cells and the exponential takes into account (harmonic) fluctuations of an individual bilayer $u_{n}$ relative to its equilibrium position $u_{o}$.

The form factor $P\left(q_{z}\right)$ contains the electron density distribution $\rho$ over the unit cell

$$
P\left(q_{z}\right)=\left|\int_{0}^{\delta} \rho_{o}(z) e^{i q_{z z}} d z\right|^{2}
$$

The electron densities of the various structural components (headgroups, hydrocarbon chains, etc.) relative to the solvent (water) are collected in Table 1 and were retrieved from the literature. ${ }^{19}$ They are independent of $\mathrm{x}$-ray measurements and have been confirmed by $\mathrm{x}$-ray data. ${ }^{20,21}$ It is assumed that the area per DPPC molecule is determined by the head group only. ${ }^{19}$ This is supported by the absence of nonlamellar phases in DPPC under excess water conditions. ${ }^{11}$ For the carbonyl group we have added 11 electrons to the 15 intrinsic due to the screening of the Na-ions in the buffer. The numbers for the mixed headgroups, i.e. carbonyl and (lyso)PC, are the sum of the two.

Our intensity simulation employs a variational approach with the carbon chain length as parameter, which is not known a priori. Figure 4 right shows the simulated intensities for the three models illustrated in Figure 4 left.

From the simulations performed, Figure 1SI,II we could exclude the single bilayers (dimers of LPC and PA intercalated, Figure 4I) and the model proposed in Figure 4II because such structures yield first order diffraction reflections with by far the highest intensities. Moreover, the second model has similar head groups facing each other, prone to repulsion. Finally, the simulation shown in Figure 1SIII, corresponds to the structure of the double-bilayer seen in Figure 4III, validating our model for the double-bilayers structure $\mathrm{L}_{\mathrm{b} \beta \mathrm{b} \beta}$ proposed. It gives a pattern with stronger second order reflection, compatible with the experimental data obtained (see other patterns shown in Figure 2).

Table 1. Parameters used and respective values of the calculated electron density profile used for the $\mathrm{x}$-ray scattering pattern simulation

\begin{tabular}{lccccc}
\hline Molecular Unit & Electrons & $\begin{array}{c}\text { Length/ } \\
\mathrm{nm}\end{array}$ & $\begin{array}{c}\text { Volume/ } \\
\mathrm{nm}^{3}\end{array}$ & $\rho$ & $\begin{array}{c}\rho_{\text {rel }} \\
\rho-\rho_{\mathrm{H} 20}\end{array}$ \\
\hline water & 10 & & $0.30^{\#}$ & 0.33 & 0.0 \\
LysoPC-head & 164 & 0.11 & $0.33^{\#}$ & 0.50 & 0.17 \\
Carbonyl head & 26 & 0.17 & 0.05 & 0.51 & 0.18 \\
Acyl chain & 120 & Variable & $0.39^{\#}$ & 0.31 & -0.02 \\
Methyl group & 9 & 0.25 & $0.05^{\#}$ & 0.17 & -0.16 \\
Mixed headgroups & 190 & 1.07 & 0.38 & 0.50 & 0.18 \\
\hline
\end{tabular}

\# data from Petrache et al. ${ }^{19}$ 


\section{DISCUSSION}

In order to explain and compatibilize both the thermodynamic and the $\mathrm{x}$-ray signature of the system, we propose the formation of a new organization for the supramolecular self-assembled mixture of equimolar LPC and PA amphiphiles. We named it a double-bilayer.

The diffraction patterns upon the lamellar-isotropic phase transition (Figure 2) are reproducible experimentally, including the scattering associated with the isotropic phase, excluding a dissociation into single mixed bilayer domains, as in pure DPPC L phase. This observation contrasts with results observed by Allegrini reporting an anisotripic liquid crystalline phase up to $55^{\circ} \mathrm{C}$, using NMR in similar mixtures. ${ }^{11}$ Looking at the DSC trace in Figure 1, one sees the first transition as a sharp endothermic peak but the second signal is much broader, spread over a wide temperature range.

Cooling from the isotropic phase leads to the formation of the double bilayer $\mathrm{L}_{\mathrm{b} \beta \mathrm{b} \beta \text {. }}$ Even above $42{ }^{\circ} \mathrm{C}$ one can already see the onset of a chain reflection whose intensity and definition rise upon further cooling, indicating improved chain ordering, until reaching the formation of the $\mathrm{L}_{\mathrm{b} \beta i \beta}$ phase, with a shorter lamellar, lattice (Figure 1). This is a metastable phase coexisting with the $\mathrm{L}_{\mathrm{b} \beta \mathrm{b} \beta}$ until it vanishes after the equilibriation at $35.5{ }^{\circ} \mathrm{C}$. Note that the reflection on the WAXS does not change position, indicating a common organization of the chains in both phases.

As we have modelled it, the $\mathrm{L}_{\mathrm{b} \beta \mathrm{i} i}$ phase is composed of two distinct bilayers - a LPC plus a PA bilayer - in direct contact, i.e. the cholines of the LPC face the carboxyl groups of the PA, see Figure 4III. The remarkable feature of such structure is the separation of each component into two homogenous bilayers that together form the double-bilayer.

Assuming all-trans configuration for both amphiphiles forming the double-bilayers and taking the lengths of LPC and PA to be 3.1 and $2.2 \mathrm{~nm}$ respectively, we can predict very well the measured spacing of both lamellar phases; The double-bilayer $\mathrm{L}_{\mathrm{b} \beta \mathrm{b} \beta}$ with $10.9 \mathrm{~nm}$ and the $\mathrm{L}_{\mathrm{b} \text { Bi }}$ with $8.5 \mathrm{~nm}$ assuming it to show interdigitation of the fatty acid chains, i.e. a PA-interdigitated double-bilayer.

The lysolipid and the palmitic acid have the same chain lengths, so the two bilayers forming the double-bilayer should have similar response to thermal activation. Moreover, the thin water regions i.e., the head group interfaces, are also equivalent; Figure 4III, contributing to the stability of this double-bilayer arrangement.

The isotropic-lamellar phase transition involves a drastic change in the topology of the mesogenic units, therefore equilibration time affects the long range order of the double-bilayer phases, improving their regularity. The lamellar $\mathrm{L}_{\mathrm{b} \beta \mathrm{b} \beta}$ phase forming upon cooling is expected to have some defects. A similiar situation should occur for the next phase to be fomed, $\mathrm{L}_{\mathrm{b} \beta \mathrm{i} i}$, but with a larger amount of defects. We observed that holding the sample for $30 \mathrm{~min}$ at $35.5^{\circ} \mathrm{C}$ in the cooling run improves the $\mathrm{L}_{\mathrm{b} \beta \mathrm{b} \beta}$ diffraction patterns resolution (Figure 3). Comparatively, Figure 1 shows that the signal from the interdigitated strucutre is very weak and the intensity does not increase upon cooling, although the signal from the non-interdigitated phase continuously decreases its intensity. In parallel, the diffuse scattering at small angles increases and can be attributed to the formation of small lamellar domains prior to their incorporation into the $\mathrm{L}_{\mathrm{b} \beta \mathrm{i} i}$ phase. This process can be followed examining Figure 1 and explains the observation of a very broad and diffuse signal in the DSC thermogram on cooling. This observation points towards a relaxation process taking place during the transition from the double bilayer to $\mathrm{L}_{\beta}$ , via the interdigitated equivalent. In both cases the long range order is not nearly as well developed as the one from the one component lipid system in excess water. One should consider that in particular the non interdigitated $\mathrm{L}_{\mathrm{b} \beta}$ component of the $\mathrm{L}_{\mathrm{b} \beta \mathrm{i} \beta}$ phase, should contain a certain degree of "gauche conformation" distributed along the whole molecule, ${ }^{11}$ although still maintaining the characteristics of a gel phase.
We consider the interdigitation of the fatty acid to be a slow process, reflecting the time necessary to build up the long range order, characteristic of both lamellar double-bilayers $\mathrm{L}_{b \beta b \beta}$ and $\mathrm{L}_{b \beta i \beta}$. Comparing the WAXS and SAXS $\left(\mathrm{L}_{\mathrm{b} \beta \mathrm{i} i}\right)$ scattering peaks can make this point clearer. The $\mathrm{L}_{\mathrm{b} \beta \mathrm{i} i} \mathrm{SAXS}$ peaks are relatively weak and broad throughout, while upon the bilayers formation one sees a quicker rising of the WAXS peak that is associated with the chain ordering common to both double-bilayer phases, see Figures 1, 3, 4III and 1S (Supplementary Material). The relative contribution of each phase can be followed examining the increase in the ratio between intensities of the peaks in the WAXS and SAXS regions, $\mathrm{I}_{\mathrm{WAXS}} / \mathrm{I}_{\mathrm{SAXS}}$, Figure $1 \mathrm{~S}$. The excess increase from the WAXS intensity represents the contribution from the non- interdigitated $\mathrm{L}_{\mathrm{b} \beta \mathrm{b} \beta}$ double bilayer.

Comparing the temperature range of changes in the WAXS d-spacing and the integral intensity of this reflection allows us to look at two independent processes. A small decrease in d-spacing reflects a thicker packing of the paraffinic chains (Figure 1) and being independent of the increasing integral intensity, associated with an improved long range order of these chains (Figure 2S), shows that the two processes are decoupled.

\section{CONCLUSION}

Preparation of LPC/PA mixtures in excess water conditions and room temperature, leads to a initial behaviour indeed similar to that of DPPC. This implies that the main transition of the LPC is overlaid by the interaction with PA which in counterpart inhibits its micellization. As a result one has the formation of the lamellar gel structure. Increasing the temperature overpowers the interaction between the components of the mixture, leading initially to the formation of the double-bilayer and later to a lamellar-isotropic phase transition. This overpowering is best seen on slow cooling, Figure 1: At the phase transition, the bilayers form and organize into the double-bilayer arrangement, setting the basis for the PA interdigitation seen upon further cooling.

To support this concept, we observe the role of curvature, e.g. rim of defects in lamellae, in fat metabolism. The formation of synapticlike microvesicles - SLMV- is affected by PLA . It was concluded that this enzyme reduce the basal SLMV formation when antagonizing the activity of endophilin 1 in the conversion of lysophosphatidic acid to phosphatidic acid, condition for the SLMV formation. ${ }^{22}$. Patton observed the formation of a lamellar phase followed by the appearance of a viscous isotropic phase, ${ }^{23}$ most likely of cubic symmetry, upon hydrolysis of emulsified fat droplets. In terms of kinetics, it has been observed that the activation rate of the PLA $_{2}$ enzyme during the initial phase increases with decreasing phospholipid vesicle size. ${ }^{24}$

We suggest that due to the strain from curvature, vesicles or micelles, can only temporarily accommodate the products of fat digestion, prior to the formation of a double-bilayer. Indeed, the formation of this phase, with very similar characteristics to the double-bilayer in LPC/PA-mixtures, due to PLA 2 activity on DPPC at $42.1{ }^{\circ} \mathrm{C}$ has been observed. ${ }^{25}$ Thus, either reaction products or a simple mixture of components yields the same phases - the formation of double-bilayer structures, $\mathrm{L}_{\mathrm{b} \beta \mathrm{b} \beta}$ and $\mathrm{L}_{\mathrm{b} \beta \mathrm{i} \beta}$. Moreover, Jain et al. have observed that

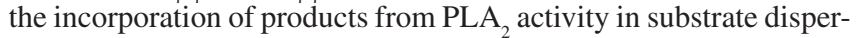
sions increases the binding affinity rather than increase the rate of binding. ${ }^{26}$ These observations were consistent with their hypothesis that pancreatic enzyme binds to defect sites at the phase boundaries in substrate bilayers induced by the PLA2 activity products

We may speculate about the biological significance of the double bilayer structure since the vast majority of organelles indeed have a two bilayer membrane. The inner and the outer membranes are known to be of different lipid composition. The double bilayer discussed here may thus be further used when a simple model of a cell membrane is required. 


\section{SUPPEMENTARY MATERIAL}

In http://quimicanova.sbq.org.br

\section{ACKNOWLEDGEMENTS}

We thank Drs V. M. Haramus and L. A. S. A. Prado for the support in the attempt to identify and characterize micelles we believed to exist at high temperatures.

\section{REFERENCES AND NOTES}

1. Lehninger, A. L.; Biochemistry, Worth Publishers Inc.: New York, 1979.

2. Jain, M. K.; Bruch, M. In Membrane Structure in Disease and Drug Therapy; Zimmer, G., ed.; Marcel Dekker: New York, 1999.

3. Andresen, T. L.; Jensen, S. S.; Kaasgaard, T.; Jørgensen, K.; Curr. Drug Delivery 2005, 2, 353.

4. Kini, R. M. In Venom phospholipase A2 Enzymes: Structure, Function and Mechanism; Kini, R. M., ed.; John Wiley \& Sons: Chichester, 1997. p. 1-28.

5. Scott D. L.; White, S. P.; Otwinowski, Z.; Yuan, W.; Gelb, M. H.; Sigler, P. B.; Science 1990, 250, 1541.

6. Thunnissen, M. M. G. M.; Eiso, A. B.; Kalk, K. H.; Drenth, J.; Dijkstra, B. W.; Kuipers, O. P.; Dijkman, R.; de Haas, G. H.; Verheij, H. M.; Nature 1990, 347, 689.

7. Justesen, P. H.; Kristensend, T.; Ebdrup, T.; Otzen, D.; J. Coll. Int. Sci. 2004, 279, 399; Hønger, T.; Jørgensen, K.; Stokes, D.; Biltonen, R. L.; Mouritsen, O. G.; Methods Enzymol. 1997, 286,168.

8. Lemmich, J.; Richter, F.; Hønger-Callisen, T.; MRS Proceedings 1998, 489,125 .

9. Jain, M. K.; van Echteld, C. J. A.; Ramírez, F.; de Gier, J.; de Haas, G. H.; van Deenen, L. L. M..; Nature 1980, 284, 486.

10. Marsh, D., ed.; Handbook of Lipid Bilayers, CRC Press: Boca Raton 1990.

11. Allegrini, P. R.; van Scharrenberg, G.; de Haas, G. H.; Seelig, J.; Biochim. Biophys. Acta 1983, 731, 448.

12. Rapp, G.; Acta Phys. Pol., A 1992, 82, 103.

13. Rapp, G.; Gabriel, A.; Dosière, M.; Koch, M. H. J.; Nucl. Instr. Meth. A, 1995, 357, 178.
14. The nomenclature proposed for this phase is based on Luzatti's nomenclature for liquid crystals [see Luzzati, V.; In Biological Membranes, D. Chapman Ed.; (Academic Press, London, 1968)] and considers the following. $\mathrm{L}$ indicates a lamellar structure. A subscript $\mathrm{b} \beta$ indicates a bilayer (b) and ( $\beta$ ) the paraffinic chains with all-trans configuration. Similarly the subscript i $\beta$ indicates a layer of interdigitated (i) paraffinic chains also with all-trans configuration $(\beta)$. The first pair is assigned to the LPC bilayer and the second to the PA (bi)layer. We would like to remark that the term double bilayer has already appeared in the literature (Bouwstra, J. A.; Thewalt, J.; Gooris, G. S.; Kitson, N.; Biochemistry 1997, 36, 7717) but not related to a lamellar structure possessing two different bilayers per unit cell.

15. Lipidat database - http://www.lipidat.ul.ie, accessed in September 2008.

16. Boyanov, A.; Tenchov, B.; Koynova, R.; Koumanov, K.; Biochim. Biophys. Acta 1983, 732, 711.

17. Our model can easily justify the formation of the double-bilayers, $\mathrm{L}_{\mathrm{b} \beta \mathrm{b} \beta}$ and its interdigitated form, $\mathrm{L}_{\mathrm{b} \beta i \beta}$. However we could not unequivocally identify which one of the $\mathrm{L}_{\text {LPC-LPC }}$ or $\mathrm{L}_{\mathrm{PA}-\mathrm{PA}}$ interdigitates. Intuitively we suggest that the $\mathrm{L}_{\mathrm{PA}-\mathrm{PA}}$ does it because of the higher melting temperature of PA compared to LPC in excess water. A determination of a well resolved electron density profile of the interdigitated double-bilayer phase requires diffraction patterns containing several orders (not available), that would allow one to securely confirm the proposed configuration.

18. Nallet, F.; Laversanne, R.; Roux, D.; J. Phys. II France 1993, 3, 487.

19. Petrache, H. I.; Feller, S. E.; Nagle, J. F.; Biophys. J. 1997, 72, 2237.

20. Nagle, J. F.; Wiener, M. C.; Biochim. Biophys. Acta 1988, 942, 1.

21. Sun, W.-J.; Suter, R. M.; Knewtson, M. A.; Worthington, C. R.; TristramNagle, S.; Zhang, R.; Nagle, J. F.; Phys. Rev. E 1994, 49, 4665.

22. Schmidt, A.; Wolde, M.; Thiele, C.; Fest, W.; Kratzin, H.; Podtelejnikov, A. V.; Witke, W.; Huttner, W. B.; Sölling, H.-D.; Nature 1999, 401, 133.

23. Patton, J. S.; Carey, M. C.; Science 1979, 204, 145.

24. Gheriani-Gruszka, N.; Almog, S.; Biltonen, R. L.; Lichtenberg, D.; J. Biol. Chem. 1988, 263, 11808.

25. Richter, F.; Estrela-Lopis, I.; Dahmen-Levison, U.; Brezesinski, G.; Rapp, G.; Biophys. J. 2000, 78, A415.

26. Jain, M. K.; Egmond, M. R.; Verheij, H. M.; Apitz-Castro, R.; Dijkman R.; de Haas, G. H.; Biochim. Biophys. Acta 1982, 688, 341. 
DOUBLE-BILAYER: A NEW PHASE FORMED BY LYSOPHOSPHOLIPIDS AND THE CORRESPONDING FATTY ACID

Sérgio S. Funari*\#

EMBL Outstation Hamburg, DESY

Gert Rapp ${ }^{\# 2}$

EMBL Outstation Hamburg, DESY

Frank Richter

EMBL Outstation Hamburg, DESY, Dept. of Physics, E22 Biophysics, TU Munich, D-85748 Garching, Germany

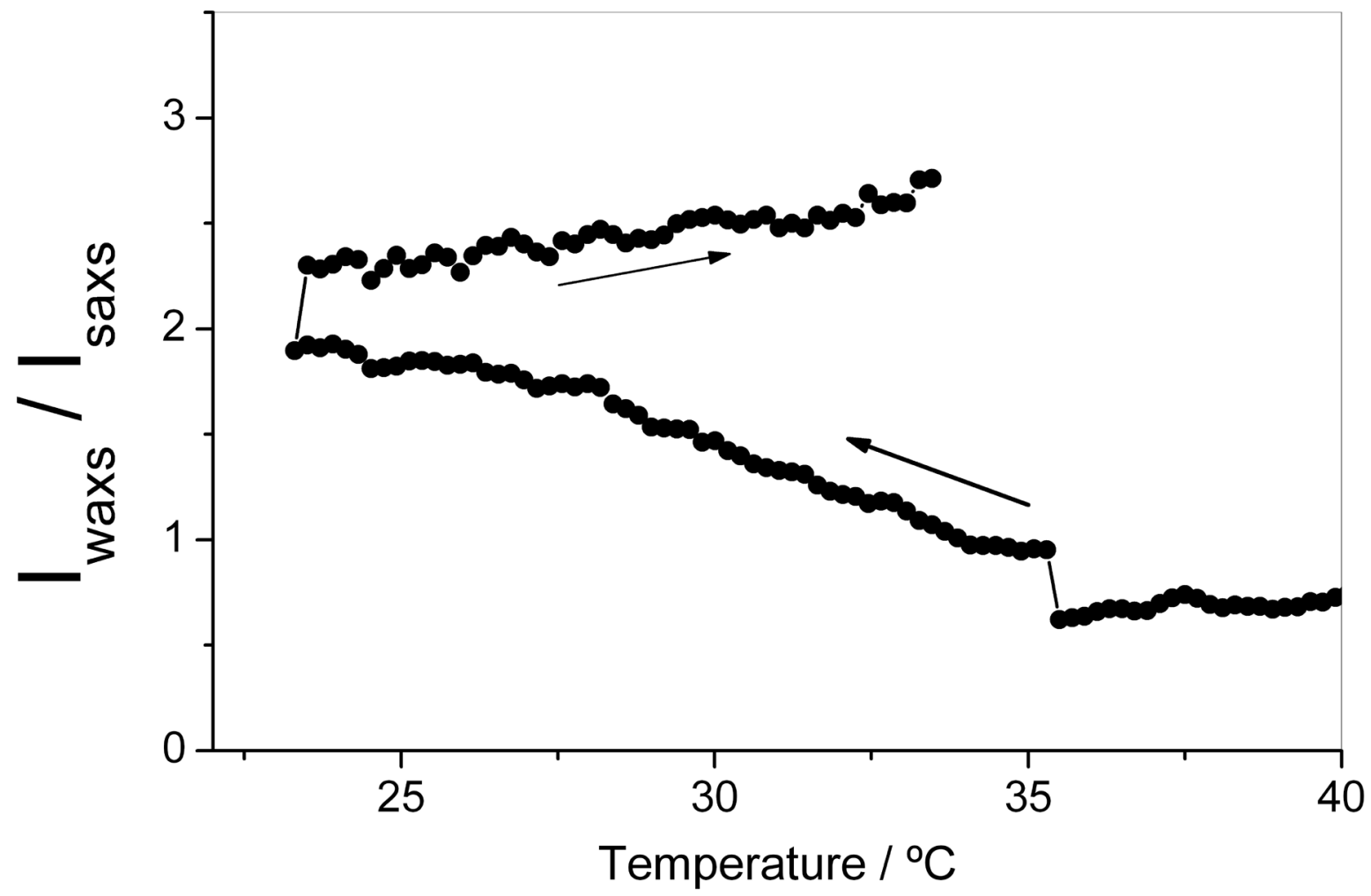

Figure 1S. Ratio between the integral intensities of the WAXS and SAXS peaks during the temperature scan. Note the effect of 30 minutes equilibration at $35.5^{\circ} C$, changing from a predominantly forming process to an ordering one. At the same time the interdigitated double-bilayer becomes visible (see Figure 1)

\#Present address: HASYLAB at DESY, D-22607, Notkestr. 85, Hamburg, Germany.

*e-mail: sergio.funari@desy.de

\#2 Present address: Rapp OptoElectronic, Gehlenkamp 9a, 22559 Hamburg,

Germany 